\title{
Multi-Frequency Encoding for Fast Color Flow or Quadroplex Imaging
}

\section{Oddershede, Niels; Gran, Fredrik; Jensen, Jørgen Arendt}

Published in:

I E E E Transactions on Ultrasonics, Ferroelectrics and Frequency Control

Link to article, DOI:

10.1109/TUFFC.2008.712

Publication date:

2008

Document Version

Publisher's PDF, also known as Version of record

Link back to DTU Orbit

Citation (APA):

Oddershede, N., Gran, F., \& Jensen, J. A. (2008). Multi-Frequency Encoding for Fast Color Flow or Quadroplex Imaging. I E E E Transactions on Ultrasonics, Ferroelectrics and Frequency Control, 55(4), 778-786.

https://doi.org/10.1109/TUFFC.2008.712

\section{General rights}

Copyright and moral rights for the publications made accessible in the public portal are retained by the authors and/or other copyright owners and it is a condition of accessing publications that users recognise and abide by the legal requirements associated with these rights.

- Users may download and print one copy of any publication from the public portal for the purpose of private study or research.

- You may not further distribute the material or use it for any profit-making activity or commercial gain

- You may freely distribute the URL identifying the publication in the public portal 


\title{
Multi-Frequency Encoding for Fast Color Flow or Quadroplex Imaging
}

\author{
Niels Oddershede, Fredrik Gran, and Jørgen Arendt Jensen, Senior Member, IEEE
}

\begin{abstract}
Ultrasonic color flow maps are made by estimating the velocities line by line over the region of interest. For each velocity estimate, multiple repetitions are needed. This sets a limit on the frame rate, which becomes increasingly severe when imaging deeper lying structures or when simultaneously acquiring spectrogram data for triplex imaging. This paper proposes a method for decreasing the data acquisition time by simultaneously sampling multiple lines for color flow maps, using narrow band signals with approximately disjoint spectral support. The signals are separated in the receiver by filters matched to the emitted waveforms, producing a number of data sets with different center frequencies. The autocorrelation estimator is then applied to each of the data sets. The method is presented, various side effects are considered, and the method is tested on data from a recirculating flow phantom. A mean standard deviation across the flow profile of $3.1,2.5$, and $2.1 \%$ of the peak velocity was found for bands at $5 \mathrm{MHz}, 7 \mathrm{MHz}$, and $9 \mathrm{MHz}$, respectively. Alternatively, the method can be used for simultaneously sampling data for a color flow map and for multiple spectrograms using different spectral bands. Using three spectral bands, data for a color flow map and two independent spectrograms can be acquired at the time normally spent on acquiring data for a color flow map only. This yields an expansion of triplex imaging called multifrequency quadroplex imaging, which enables study of the flow over an arterial stenosis by simultaneously acquiring spectrograms on both sides of the stenosis, while maintaining the color flow map. The method was tested in vivo on data from the common carotid artery of a healthy male volunteer, both for fast color flow mapping and for multifrequency quadroplex imaging.
\end{abstract}

\section{INTRODUCTION}

CINCE the introduction of the autocorrelation estimator for ultrasonic blood flow imaging by Kasai et al. [1] in the mid-1980s, it has been possible to create full color flow maps (CFM) in real time. The method is robust and fairly simple to implement. The velocities are estimated line by line over the region of interest, and for each line, repeated transmissions are needed. The performance of the estimator is closely linked to the number of repetitions used to form the estimate [2]. An inherent trade-off between frame rate and performance therefore exist. When imaging deep

Manuscript received May 3, 2007; accepted December 8, 2007. This work was supported by grant 26-04-0024 from the Danish Science Foundation, by the Danish research council for Technology and Production grant 274-05-0327, and by B-K Medical A/S, Herlev, Denmark.

The authors are with the Center for Fast Ultrasound Imaging, Ørsted•DTU, Technical University of Denmark, DK-2800 Kgs. Lyngby, Denmark (e-mail: no@oersted.dtu.dk).

Digital Object Identifier 10.1109/TUFFC.2008.712 structures such as the heart, this might severely affect either the performance or the frame rate. Also, in the triplex imaging mode where both CFM data and a spectrogram are shown, the time for data acquisition must be divided between multiple sets of data, increasing the significance of this problem even further.

The autocorrelation estimator essentially assumes a narrow-band signal, and the variance of the velocity estimates decreases as the bandwidth is decreased [2]. Therefore, a long sinusoidal narrow-band pulse is used for CFM. Nevertheless, ultrasound transducers are often designed fairly wide-band to ensure good resolution in B-mode images. Using only a narrow frequency band for collecting CFM data does not exploit the available bandwidth efficiently.

Numerous wide-band estimators have been proposed for ultrasonic blood velocity estimation including crosscorrelation [3], maximum likelihood estimation [4], twodimensional Fourier transformation methods [5], [6], among others. Common for all these methods is increased computational complexity, which is probably the reason why the autocorrelation approach is still the most used estimator in commercial equipment. Use of the wide band of the transducer has also been proposed for improving the velocity estimates in the autocorrelation approach by emitting a broad-band pulse [7]. The received signals are then filtered into numerous narrow bands, and the velocity is estimated in each band. The velocity estimates can then be combined to form a better estimate [8] by, for instance, averaging. Still, none of these methods directly address the limitation set by the time needed for data acquisition.

Parallel receive beamforming has been proposed for decreasing the time spent on data acquisition for B-mode images [9]. Here a broadly focused transmit beam is emitted, and multiple receive beams are generated simultaneously by steering the beams in slightly different directions. This method has also been investigated for blood flow estimation applications [10].

This paper proposes a method for significantly decreasing the time spent on data collection for CFM by simultaneously sampling multiple lines using different frequency bands. The signals are then separated in the receiver by a simple filtering operation and the autocorrelation estimator is applied. The number of frequency bands $M$ used depends on the available transducer bandwidth and the intensity limits set by the Food and Drug Administration [11]. The total time spent on CFM data acquisition will decrease by a factor of $M$, potentially increasing the frame rate by a factor of $M$. Alternatively, the proposed 
method can be used for simultaneously acquiring CFM data and spectrogram data for triplex imaging. This paper will demonstrate how data for an extension of triplex imaging, namely, multi-frequency quadroplex (MFQ) imaging featuring two independent spectrograms and a CFM, can be acquired during the time normally spent on acquiring a CFM.

While the parallel receive beamforming presented in [9] is limited to sampling of closely spaced lines within the transmitted beam, the proposed method provides a larger flexibility in where data are sampled. Ultimately, the two methods could be combined, yielding either the possibility of a very high frame rate of CFMs, or introducing the possibility of making CFMs of 3D volumes at an acceptable frame rate.

Simultaneously transmitting multiple frequency bands in ultrasound imaging from different spatial locations and separating the signals in the receiver is not a new concept. It has previously been used for frequency division in synthetic transmit aperture imaging, where a broadband pulse is synthesized using multiple narrow-band signals [12], and for directional velocity estimation in synthetic transmit aperture ultrasound [13]. In this paper, no broad-band synthesis is performed, but the narrow-band signals are essentially used directly in the autocorrelation estimator and for spectral estimation.

The remainder of the paper is organized as follows. Section II gives a short review of the autocorrelation method and spectral velocity estimation and describes the proposed encoding method. In Section III, the proposed method is tested quantitatively in a flow phantom, both for simultaneous sampling of multiple CFM lines and for MFQ imaging, and Section IV presents in vivo results from the common carotid artery of a healthy male volunteer. The method is finally discussed in Section V.

\section{THEORY}

\section{A. Autocorrelation Estimator}

The autocorrelation method for ultrasonic blood flow imaging was first introduced by Kasai et al. [1]. Narrowband pulses are repeatedly emitted along the same direction, and the received RF data are Hilbert transformed to give the in-phase and quadrature component. This results in a complex matrix $y(l, i)$, where $i$ is the transmission number and $l$ is the sample index along the RF line corresponding to depth $d_{l}=\frac{c}{2 f_{s}} l$. The axial velocity at a given depth can be estimated as [1]

$$
v_{z}=-\frac{c f_{\text {prf }}}{4 \pi f_{0}} \arctan \left(\frac{\Im\{R(1)\}}{\Re\{R(1)\}}\right),
$$

where $c$ is the speed of sound, $f_{\text {prf }}$ is the pulse repetition frequency, $f_{0}$ is the center frequency of the emitted narrow-band signal, and $R(1)$ is the complex autocorrelation function of $y(l, i)$ at lag 1 , evaluated at a certain depth corresponding to $l=L_{d}$. This autocorrelation function can be estimated by

$$
\hat{R}(1)=\frac{1}{(N-1) N_{l}} \sum_{l=0}^{N_{l}-1} \sum_{i=0}^{N-2} y\left(l+L_{d}, i\right) y^{*}\left(l+L_{d}, i+1\right)
$$

which includes an averaging over $N_{l}$ RF samples. This has been shown to lower the variance of the estimated autocorrelation function, and thereby increase the accuracy of the velocity estimate [14].

By demanding that a whole period of the signal be observed to distinguish the flow signal from that of a stationary structure, the minimum detectable velocity of an autocorrelation estimator is given by [2]

$$
v_{\min }=\frac{c}{2} \frac{f_{\mathrm{prf}}}{N f_{0}}
$$

This is a quite conservative demand, and $v_{\min }$ is not considered a rigid limit. According to the Nyquist sampling theorem, the maximum detectable velocity of an autocorrelation estimator is [2]

$$
v_{\max }=\frac{c}{2} \frac{f_{\mathrm{prf}}}{2 f_{0}+B}
$$

where $B$ is the bandwidth of the emitted signal. The variance of the velocity estimate, assuming a constant velocity, and under the assumption that no noise is present, can be approximated by [2]

$$
\sigma_{v}^{2}=\frac{c}{4 \pi^{2} f_{0}^{2}} \frac{f_{\mathrm{prf}}}{T}\left|v_{z}\right|
$$

where $T$ is the duration of the emitted pulse.

\section{B. Spectral Velocity Estimation}

A spectrogram displays variation of the spectral content of the slow-time signal over time, thereby yielding a direct measure of the axial velocity of the moving blood. It can be estimated from the complex signal matrix $y(l, i)$ when the number of observations is sufficiently high. At a certain time instance $t=k / f_{\text {prf }}$, the power spectrum over pulse repetitions is estimated from $N_{s}$ pulse repetitions and averaged over a number of RF samples $N_{l}$, which is known as the range gate. The segment size $N_{s}$ is chosen low enough to capture the frequency variations over time and high enough to give an acceptable spectral resolution. A window $w(i)$ is often applied to the data prior to the Fourier transform. The estimated power spectral density is given by

$$
\begin{aligned}
& \hat{P}_{y}\left(f_{p}, k\right)= \\
& \frac{1}{N_{l}} \frac{1}{N_{s}} \sum_{l=0}^{N_{l}-1}\left|\sum_{i=0}^{N_{s}-1} w(i) y\left(l+L_{d}, i+k\right) e^{-j 2 \pi f_{p} i / f_{\mathrm{prf}}}\right|^{2},
\end{aligned}
$$




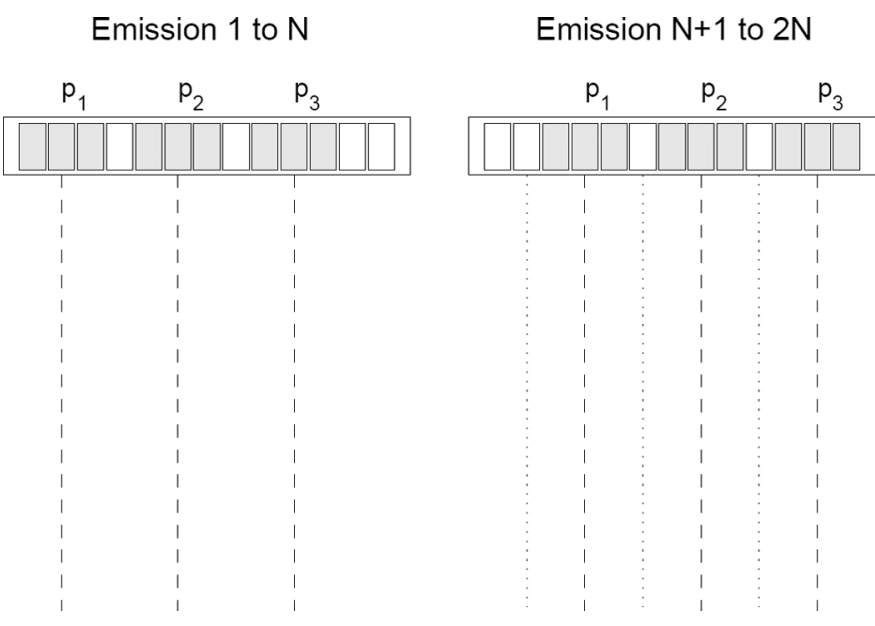

Fig. 1. The basic principle of the proposed method. Three different pulses $p_{1}(t), p_{2}(t)$, and $p_{3}(t)$ are emitted $N$ times and the received signals are separated in the receiver to simultaneously create three lines of the color flow map. The position of the transmitting subapertures are shifted and new lines are created.

which, for a certain depth corresponding to RF index $L_{d}$, is a function of frequency $f_{p}$ and time given by pulse repetition number $k$. Using a Hamming window given by

$$
w(i)=0.54-0.46 \cos \left(\frac{2 \pi i}{N-1}\right), \quad i=0,1, \ldots, N-1
$$

will lower the spectral side-lobes, coming from the limited observation time, to approximately $-40 \mathrm{~dB}$.

The estimated power spectrum is displayed as brightness on a logarithmic scale, with the frequency axis vertically and the time axis horizontally. The frequency axis is often scaled to give the axial velocity estimate through

$$
v_{z}=\frac{c}{2 f_{0}} f_{p}
$$

visualizing the changes in the axial velocities within the range gate over time. A spatial velocity distribution within the range gate will result in a broadening of the power spectrum [1]. Due to the sampling of the slow-time signal with sampling frequency $f_{\text {prf }}$, the power spectrum $\hat{P}_{y}\left(f_{p}, k\right)$ has repetitions at all integer multiples of $f_{\text {prf }}$.

\section{Spatial Encoding Using Frequency Division}

The proposed method aims at increasing the frame rate of color flow mapping by simultaneously sampling data for multiple lines in the CFM. Fig. 1 (left) shows a linear array transducer simultaneously emitting $M=3$ different pulses $p_{1}(t), p_{2}(t)$, and $p_{3}(t)$ using different subapertures. If it is assumed that the signals can be separated in the receiver, three different lines can be beamformed after each emission. The emission is repeated $N$ times at a given pulse repetition frequency $f_{\text {prf }}$, and three lines in the CFM are created using the autocorrelation estimator [1]. The transmitting subapertures are slid and the emissions are repeated until data for the entire CFM are collected. The time used for collecting data will, in this example, be only a third of that normally used.

Alternatively, the CFM data can be acquired as is usually done, using one narrow-band signal $p_{1}(t)$. Simultaneously, data for two spectrograms can be acquired using the signals $p_{2}(t)$ and $p_{3}(t)$. These signals are repeatedly emitted from the same subapertures, continuously sampling along the same lines. Thus, a CFM and two spectrograms are acquired simultaneously, yielding an expansion of triplex imaging called multi-frequency quadroplex (MFQ) imaging. The data acquisition time will equal that normally spent on acquiring a CFM. At some point, a transducer element will be required to emit both a delayed version of $p_{1}(t)$ and, for instance, $p_{2}(t)$. To use the entire amplitude range while emitting each signal, $p_{2}(t)$ and $p_{3}(t)$ are delayed so that they are emitted later than $p_{1}(t)$.

The emitted signals $p_{1}(t), p_{2}(t)$, and $p_{3}(t)$ are designed as simple narrow-band pulses at different frequencies. They are given by

$$
p_{m}(t)=w(t) \sin \left(2 \pi f_{m} t\right), \quad 0<t<T
$$

for $m=[1,2,3]$, where $f_{m}$ is the center frequency of the $m$ th signal, and $T$ is the pulse duration. The term $w(t)$ is a window designed to reduce the spectral leakages into the other bands. A window giving good spectral side-lobe suppression is the Hamming window,

$$
w(t)=0.54-0.46 \cos \left(\frac{2 \pi t}{T}\right), \quad 0<t<T,
$$

which has spectral side-lobes below $-40 \mathrm{~dB}$. In the receiver, the signals are then separated by filters matched to the emitted signals

$$
h_{m}(t)=p_{m}(T-t), \quad 0<t<T .
$$

Other more advanced design methods could, of course, be used for designing the signals and filters. This will be considered in Section II-D. The number of pulses $M$, which can be emitted, is determined by the transducer bandwidth compared to the bandwidth of the signals $p_{m}(t)$. Fig. 2 shows the transfer function of a commercial linear array transducer (thick line), and the amplitude spectra of three signals $p_{1}(t), p_{2}(t)$, and $p_{3}(t)$, using $f_{m}=$ $[5,7,9] \mathrm{MHz}$ and $T=2 \mu \mathrm{s}$ (thin lines). The spectral leakage is below $-40 \mathrm{~dB}$ for all bands.

The minimum detectable velocity is now limited by the lowest frequency band, centered at $f_{1}$,

$$
v_{\text {min }}=\frac{c}{2} \frac{f_{\mathrm{prf}}}{N f_{1}},
$$

and the maximum detectable velocity is limited by the highest frequency band $f_{M}$,

$$
v_{\max }=\frac{c}{2} \frac{f_{\mathrm{prf}}}{2 f_{M}+B} .
$$






Fig. 2. The two-way system transfer function and the amplitude spectra of the three emitted pulses.
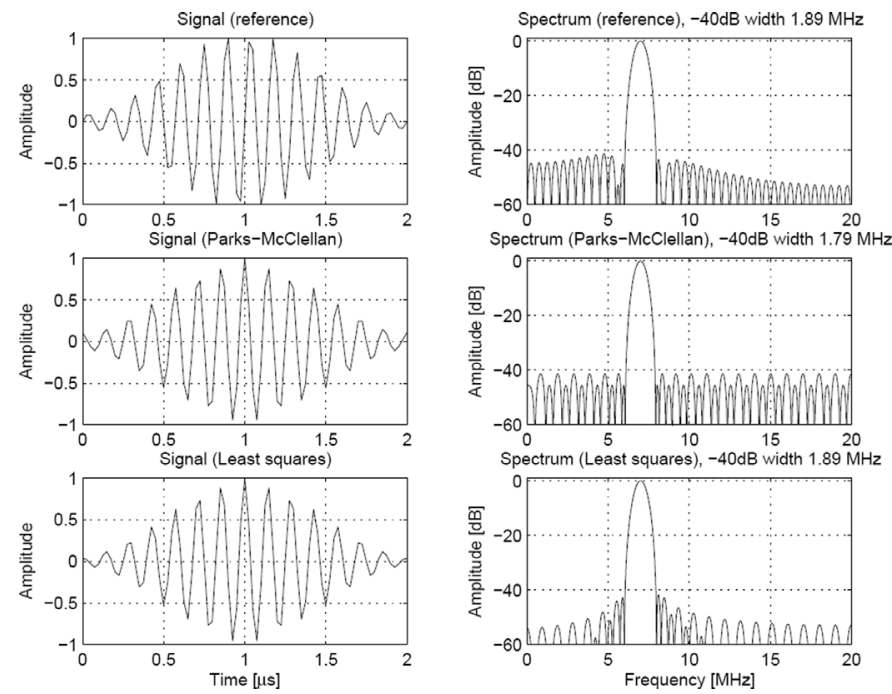

Fig. 3. A comparison of the signal $p_{2}(t)$ in (9) to a Parks-McClellan design and a least squares design. The left column shows the signals in the time domain, while the right column shows them in the frequency domain. The top row shows the signal $p_{2}(t)$, the middle row shows the Park-McClellan design, and the bottom row shows the least squares design.

The increased frame rate, thus, comes at the expense of a slightly decreased velocity range compared to a single narrow-band pulse. This will be further elaborated on in Section V. Furthermore, the method requires $M$ parallel beamformers, which are incorporated in most commercial high-end scanners at present, and a matched filtration of the channel RF data. This will also be addressed in Section V.

\section{Signal and Filter Design}

To quantify the design of the signals $p_{m}(t)$ and the corresponding matched filters $h_{m}(t)$, the $p_{2}(t)$ signal is compared to a Parks-McClellan design [15] and least squares design [16] in Fig. 3. The left column shows the signals in the time domain, while the right column shows them in the frequency domain. The signal duration was maintained at $T=2 \mu \mathrm{s}$, the center frequency at $f_{2}=7 \mathrm{MHz}$, and the maximum side-lobe level at $-40 \mathrm{~dB}$, while the $-40 \mathrm{~dB}$ bandwidth was minimized. The signal given by (9) and the least squares design both had a bandwidth of $1.89 \mathrm{MHz}$, while a slight improvement was attained us-

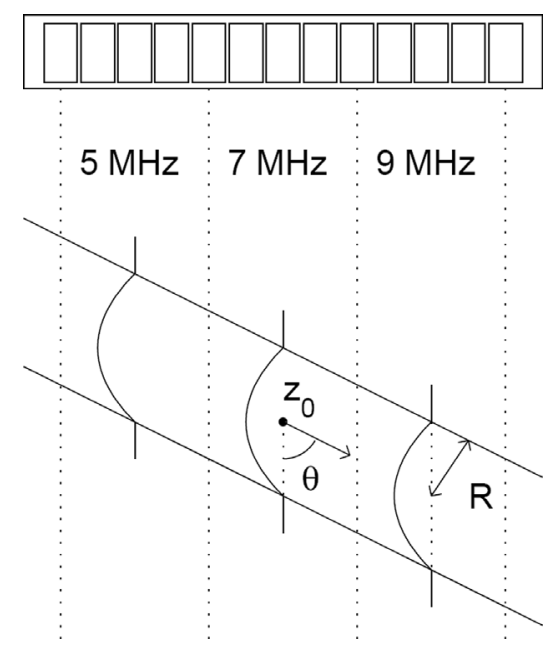

Fig. 4. Physical setup of the phantom experiment. The transducer is mounted at height $z=z_{0}$ over the tube center at an angle of $\theta=60^{\circ}$. The tube has radius $R$. The figure also shows the three regions of the CFM made at different frequency bands. Each region is sampled left to right and all regions are sampled simultaneously. The position of the three velocity profiles correspond to the profiles shown in Fig. 5.

ing the Parks-MacClellan design, where a $-40 \mathrm{~dB}$ bandwidth of $1.79 \mathrm{MHz}$ was seen. This comes at the expense of increased far side-lobes compared to the reference. The signals given by (9) are therefore used in all of the measurements presented in this paper.

\section{Phantom Experiments}

\section{A. Simultaneous Sampling of Multiple CFM Lines}

The method was tested in a recirculating flow rig, where a blood mimicking fluid was pumped at a constant velocity through a rubber tube submerged in a water tank. The tube had an internal radius of $R=6 \mathrm{~mm}$. The fluid passed a 1 meter long straight metal tube with the same radius prior to entering the rubber tube, and the Reynolds number was kept below 300, both to ensure a parabolic flow profile. The transducer was mounted in the water tank with the transducer surface $z_{0}=33.5 \mathrm{~mm}$ above the tube center and with a $\theta=60^{\circ}$ angle between the beam axis and the flow direction. The axial component of the velocity $v_{z}(z)$ at the center axis of the transducer was assumed to be parabolic and centered at $z=z_{0}$. It is given by

$$
\begin{aligned}
& v_{z}(z)=\left(1-\frac{\left(z-z_{0}\right)^{2}}{(R / \sin \theta)^{2}}\right) v_{0} \cos \theta, \\
& z_{0}-\frac{R}{\sin \theta}<z<z_{0}+\frac{R}{\sin \theta},
\end{aligned}
$$

where $v_{0}=0.1 \mathrm{~m} / \mathrm{s}$ is the peak velocity along the flow direction. The physical setup of the phantom experiment is shown in Fig. 4, which also shows the definition of the depth $z_{0}$, the tube radius $R$, and the beam-to-flow angle $\theta$.

The data acquisition was performed using a 128-element linear array transducer and the RASMUS multi-channel 
TABLE I

Parameters Used for the Phantom Experiment.

\begin{tabular}{ll}
\hline Parameter & Value \\
\hline Transducer type & Linear array \\
Number of transducer elements & 128 \\
Transducer element pitch & $0.26 \mathrm{~mm}$ \\
Transducer element height & $4 \mathrm{~mm}$ \\
Elevation focus & $15 \mathrm{~mm}$ \\
Transfer function & See Fig. 2 \\
Transmit focus depth & $28.7 \mathrm{~mm}$ \\
Number of transmit bands, $M$ & 3 \\
Center frequencies, $f_{1}, f_{2}$, and $f_{3}$ & 5,7, and $9 \mathrm{MHz}$ \\
Pulse duration, $T$ & $2 \mu \mathrm{s}$ \\
Amplitude tapering & Hamming window \\
Number of emit. elements/band & 22 \\
Transmit apodization & Tukey window \\
Number of receiving elements & $128(2 \times 64$ through \\
& multiplexing) \\
Receive apodization & Hanning window (over 64 \\
& elem. cnt. around the \\
Sampling frequency, $f_{s}$ & current image line) \\
Pulse repetition frequency, $f_{\text {prf }}$ & $40 \mathrm{MHz}$ \\
& $1.65 \mathrm{kHz}(3.3 \mathrm{kHz}$ incl. \\
Number of shots per estimate, $N$ & multiplex) \\
Clutter filtering & Subtracting mean of $N$ \\
& signals \\
Number of CFM lines created & 33 \\
Inter-line spacing & $0.52 \mathrm{~mm}$ \\
Repetitions of entire sequence & 17 \\
Internal tube radius & $6 \mathrm{~mm}$ \\
Depth to tube center & $33.5 \mathrm{~mm}$ \\
Peak velocity & $0.1 \mathrm{~m} / \mathrm{s}$ \\
Flow angle $\theta$ (w.r.t. depth axis) & $60^{\circ}$ \\
\hline & \\
& \\
&
\end{tabular}

sampling system [17]. This system can emit arbitrary waveforms on 128 elements, and sample 64 transducer elements in real-time. To acquire data from all 128 elements, the emission is repeated and multiplexing is used. This effectively halves the frame rate. The three signals shown in Fig. 2 were used, and the parameters for the experiment are given in Table I.

The acquired data were processed as described in Section II. A total of 17 repetitions of the entire sequence were processed, yielding 17 full color flow maps. The velocity profiles at the center line of each of the three regions (see Fig. 4) were extracted, and the means and standard deviations over the repetitions were found. These are shown in Fig. 5 along with the expected velocity profile given by (14).

Fig. 5 reveals a slightly higher standard deviation in the lower frequency bands. The standard deviations of the velocity estimates are averaged over the tube diameter and are given in Table II as percent of the theoretical peak velocity of $v_{0}=0.1 \mathrm{~m} / \mathrm{s}$. The increase in standard deviation, although present, is not as large as predicted by (5). For this phantom experiment performed in water, a quite good signal-to-noise ratio is attained, and no frequencydependent attenuation is present. The increased standard deviation at lower frequency bands is therefore qualita-
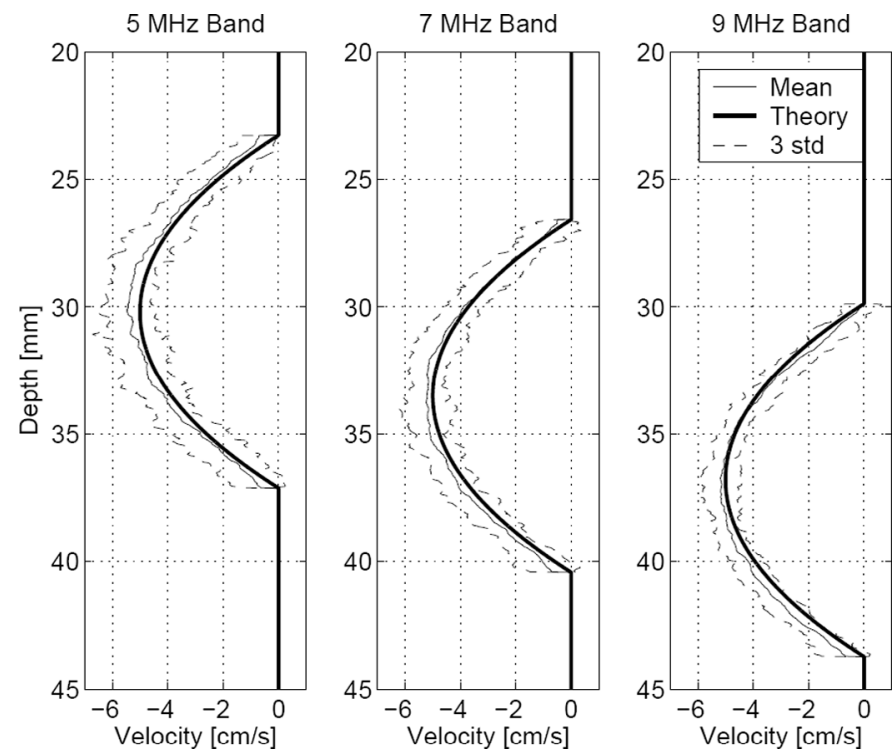

Fig. 5. Three resulting velocity profiles made at different frequency bands. The plot shows the expected velocity profile (thick line), the average of 17 profiles (thin line), and three times the standard deviation of the 17 profiles (dashed line).

TABLE II

The Average Standard Deviation of the Velocity Estimates Made at Different Frequency Bands.

\begin{tabular}{cc}
\hline Frequency band & Mean standard deviation (\%)* \\
\hline $5 \mathrm{MHz}$ & 3.1 \\
$7 \mathrm{MHz}$ & 2.5 \\
$9 \mathrm{MHz}$ & 2.2 \\
Total & 2.6 \\
\hline
\end{tabular}

* The standard deviations are given in \% of the true peak velocity $v_{0}=0.1 \mathrm{~m} / \mathrm{s}$.

tively in accordance with (5), which is derived assuming no noise.

\section{B. Multi-Frequency Quadroplex Imaging}

At present, triplex imaging is possible only at very low frame rates due to the switching between acquisition of CFM data and spectrogram data. The proposed method can be used to solve this problem. By acquiring data for the CFM in one frequency band and simultaneously acquiring the spectrogram data in another frequency band, this switching is no longer needed. If all three bands shown in Fig. 2 are used, a CFM can be created while data from two range gates are simultaneously acquired. This yields an MFQ image. Fig. 6 shows an MFQ image made in the flow rig described in Section III-A. The CFM in the top plot was made from the $5-\mathrm{MHz}$ band using $N=16$ repetitions per line (32 including multiplexing), while the leftmost spectrogram was made using the $7-\mathrm{MHz}$ band, and the rightmost spectrogram using the $9-\mathrm{MHz}$ band. The transmit focus was set at $30 \mathrm{~mm}$, a peak velocity of $v_{0}=15 \mathrm{~cm} / \mathrm{s}$ was used, and the pulse repetition frequency was set at 
$f_{\text {prf }}=1.2 \mathrm{kHz}$. The remaining parameters were set as given in Table I. The spectrograms in Fig. 6 were made in a 1-mm range gate using $N_{s}=64$ hamming weighted pulse repetition samples per estimate. The processing was done as described in Section II-B. The expected axial peak velocity was $v_{z}=v_{0} \cos (\theta)=7.5 \mathrm{~cm} / \mathrm{s}$.

\section{In VIVo EXPERIMENTS}

\section{A. Simultaneous Sampling of Multiple CFM Lines}

The method was also tested for in vivo applications. Approximately 1.5 seconds of data from the common carotid artery of a healthy 31-year-old male were acquired. The parameters of Table I were used except the depth of focus was set at $15 \mathrm{~mm}$, and the pulse repetition frequency $f_{\text {prf }}$ was set at $12 \mathrm{kHz}$ ( $24 \mathrm{kHz}$ including multiplexing), giving a maximum detectable velocity of $v_{\max }=0.46 \mathrm{~m} / \mathrm{s}$. The emitted signals were those shown in Fig. 2 and the different narrow-band signals were used in regions as shown in Fig. 4. After acquisition of each flow data frame, a B-mode image was acquired in order to create complete color flow maps.

Fig. 7 shows a frame from diastole. No post-processing is applied to the velocity estimates. The flow has a parabolic tendency and no boundary effect is seen between the three regions operating at different center frequencies. A few erroneous estimates are seen in the highest frequency band (right part). Since a larger attenuation will be experienced for the higher frequency bands, this could be a result of decreased SNR.

Fig. 8 shows a frame from systole. Again, no distinct boundaries are seen but the highest frequency band seems to display a slightly degraded performance compared to the lower frequency bands. Nevertheless, due to the order of data acquisition, boundaries will be seen during acceleration. Fig. 9 shows a frame during the early systolic accelerating phase. Here the boundaries are clearly visible.

\section{B. Multi-Frequency Quadroplex Imaging}

The MFQ imaging was also tested in vivo using a pulse repetition frequency of $f_{\mathrm{prf}}=12 \mathrm{kHz}$ (24 kHz including multiplexing) and a transmit focus depth of $15 \mathrm{~mm}$. Two examples from the common carotid artery are shown in Fig. 10 and Fig. 11 during systole and diastole, respectively. Also here, the CFM data are acquired using the 5$\mathrm{MHz}$ band, while the leftmost spectrogram is made using the $7-\mathrm{MHz}$ band, and the rightmost using the $9-\mathrm{MHz}$ band. The emission of the spectrogram waveforms is slightly delayed in time, as described in Section II-C. Thereby the same transducer element can be used for both emitting the CFM pulse (first, at $5 \mathrm{MHz}$ ) and the spectrogram pulse (second, at 7 or $9 \mathrm{MHz}$ ) at full amplitude range. The spectrogram data can therefore not be sampled at the very bottom of the image and the CFM data cannot be sampled at the very top of the image. The ordering could, of course, be interchanged if desired. The CFM is composed of 33 lines, each sampled 16 times (32 including multiplexing), and the B-mode image is made from 65 emissions. This gives a frame rate of $21.4 \mathrm{~Hz}$. Each spectrogram estimate was made from 64 lines using the method described in Section II-B. While the B-mode data were acquired (which happens 21.4 times each second), no spectrogram data were available. This is a result of the B-mode emissions using the entire transducer bandwidth. In Fig. 10, this is seen as gaps in the spectrogram. These gaps are not inherent for the proposed method, but are dependent on how the emissions are ordered. There are multiple ways of avoiding these gaps. One is to interleave the B-mode emissions and the flow data emissions, which decreases the effective pulse repetition frequency by a factor of 2. Another method was proposed by Kristoffersen and Angelsen [18], where a synthetic data segment was created, using filtering of white noise by a filter generated from the spectrogram data. Finally, the power spectrum can be estimated through the Fourier transform of the autocorrelation, which can be estimated from sparse data sequences [19].

The MFQ imaging mode yields the possibility of studying the change in flow over an arterial stenosis by placing a range gate at both sides of the stenosis and monitoring the flow over time while still maintaining the CFM. An almost two-second movie has been made from this data set where the MFQ is created at a frame rate of $21.4 \mathrm{~Hz}$. If receive multiplexing was not needed (if all 128 receiving elements could be sampled simultaneously), an MFQ with a frame rate of $42.8 \mathrm{~Hz}$ would be possible for the current region of interest.

\section{Discussion}

The simultaneous sampling of multiple lines using different frequency bands was described in Section II-C assuming the use of three narrow-band signals. The number of bands used depends on both the available transducer bandwidth and the intensity limits set by the Food and Drug Administration. When simultaneously sampling $M$ different frequency bands, the time spent on data acquisition will be $1 / M$ times the time normally spent. But the method results in a number of side effects, which must be considered.

First, the method requires an increase in hardware complexity. In order to beamform $M$ lines at each emission, $M$ parallel beamformers are needed, and the emission of multiple beams simultaneously in the transmitting frontend must be supported. Since most commercial high-end scanners at present have multiple parallel beamformers, the problem is already partly solved. Furthermore, the matched filters have to be applied directly to the sampled channel RF data in order to separate the $M$ signals. In other words, the method translates a physical problem related to the propagation speed of sound into a problem of increased processing complexity. While the speed of sound 


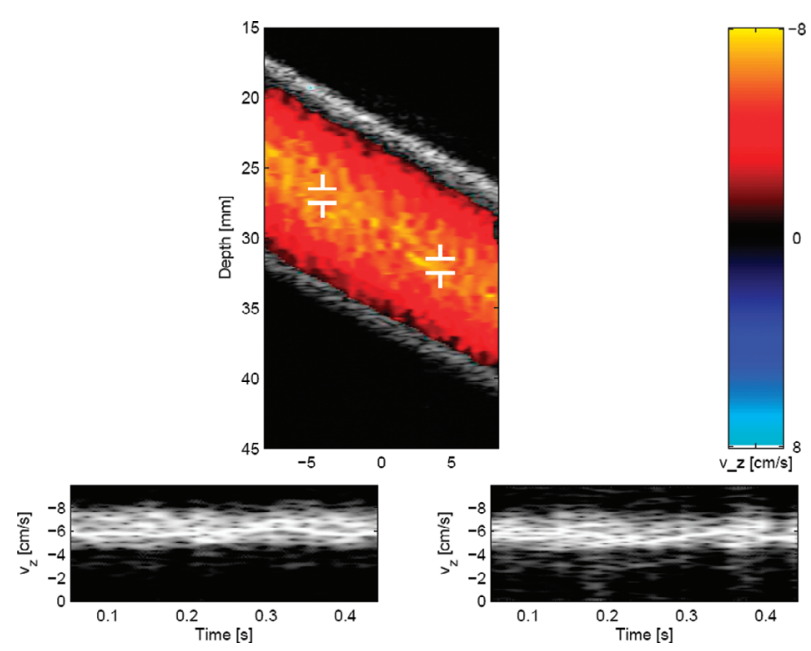

Fig. 6. Multi-frequency quadroplex image from the flow rig. The upper image shows the color flow map using the $5-\mathrm{MHz}$ band, the left spectral Doppler shows the $7-\mathrm{MHz}$ band using the leftmost range gate, and the right spectral Doppler shows the $9-\mathrm{MHz}$ band using the rightmost range gate.
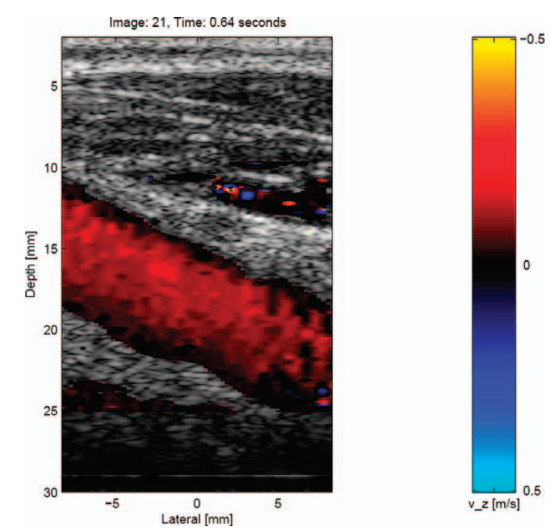

Fig. 7. Color flow map of the common carotid artery at diastole, made using the proposed method.
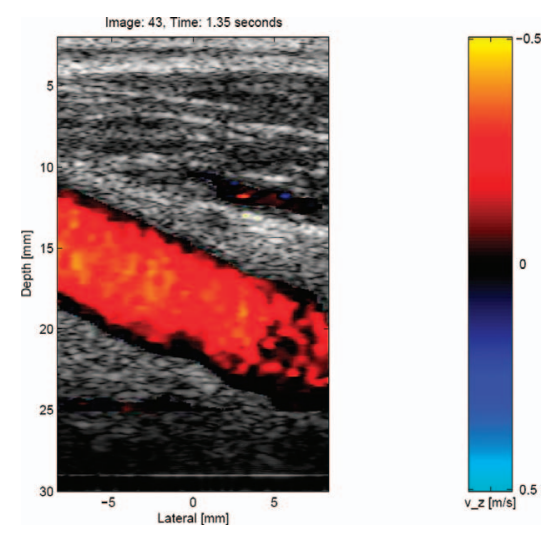

Fig. 8. Color flow map of the common carotid artery at systole, made using the proposed method.
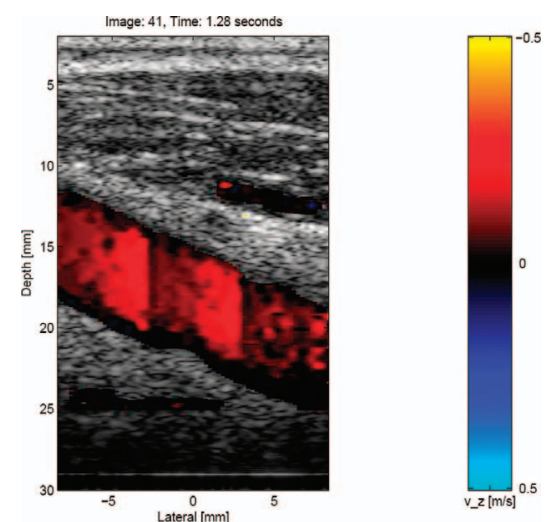

Fig. 9. Color flow map of the common carotid artery during acceleration, made using the proposed method.



Fig. 10. Multi-frequency quadroplex image of the common carotid artery during systole. The upper image shows the color flow map using the $5-\mathrm{MHz}$ band, the left spectral Doppler shows the $7-\mathrm{MHz}$ band using the leftmost range gate, and the right spectral Doppler shows the $9-\mathrm{MHz}$ band using the rightmost range gate.

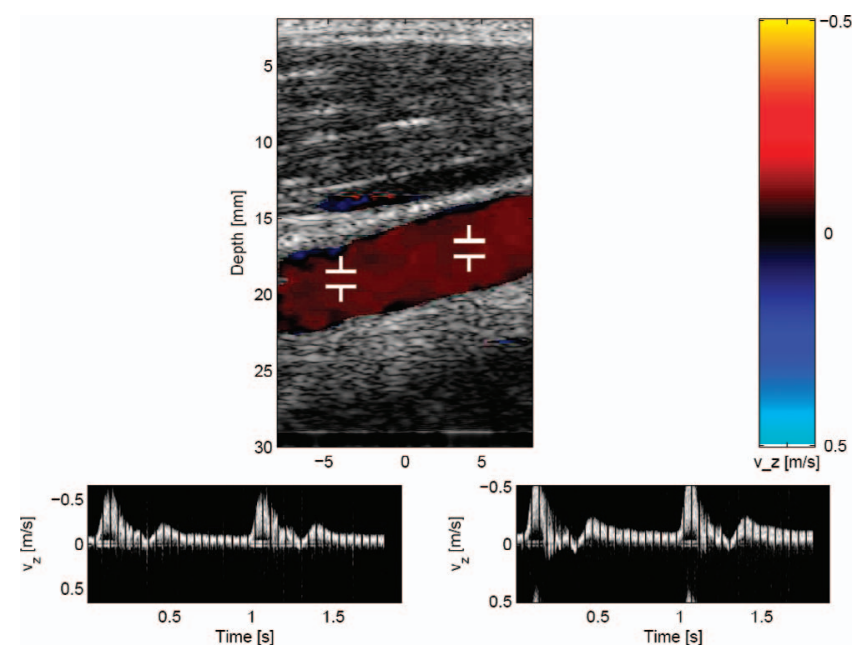

Fig. 11. Multi-frequency quadroplex image of the common carotid artery during diastole. 
$c$ is a constant, processing capability has historically increased exponentially.

Second, the performance of the velocity estimator is dependent on the center frequency of the narrow band as given by (5). This states that for the noiseless case, the variance decreases with $1 / f_{0}^{2}$. The variance at lower bands will, thus, be larger than that at higher bands, assuming no noise. At the same time, the frequency-dependent attenuation will lower the signal-to-noise ratio at higher frequency bands, which result in decreased performance of the autocorrelation estimator at higher frequency bands. The two mechanisms essentially work against each other and, depending on the depth of investigation and the type of tissue, one band will show better performance than the other.

Third, the range of velocities that can be estimated depends on the center frequency of the emitted pulse as given by (3) and (4). When using multiple frequency bands, the minimum detectable velocity will be determined by the lowest frequency band as given by (12), and the maximum detectable velocity will be given by the aliasing limit of the highest frequency band as in (13). This sets additional limits on the velocity range, and must be considered when the number of frequency bands are chosen and the emitted signals are designed. However, there is a way to work around this limitation. In commercial scanners, a filter is often applied to the velocity estimates prior to display. This could, for instance, be a median filter to remove erroneous estimates. If the frequency bands are switched when the subapertures are slid (going from the left part to the right part of Fig. 1), consecutive CFM lines are made at different frequencies. By applying a median filter of lateral size $M$ to the velocity estimates, erroneous estimates generated by a single frequency band could be removed. If we, for instance, consider the example of $M=3$ frequency bands, it would not matter if the lowest frequency band drowns in clutter or there is aliasing in the highest frequency band. This also ensures homogenous appearance of the CFM in spite of the difference of performance in the various frequency bands.

The acceleration artifact seen in Fig. 9 was a result of the acquisition order chosen for the experiment, which can be seen in Fig. 1. The lines could be acquired in the usual order by overlapping the transmitting subapertures and transmitting the waveforms slightly delayed in time, as was described in Section II-C for the MFQ mode. The CFM could then not be made at the very top and the very bottom of the imaging area, but the acceleration artifact would not appear.

Fourth, since multiple signals are emitted from the transducer simultaneously, special care must be taken to stay within the intensity limits set by the Food and Drug Administration [11]. In cases where the spatial peak temporal average intensity $I_{\mathrm{SPTA}}$ or the transducer surface temperature sets the limit of the system, it might be necessary to slightly reduce the transmit voltage, affecting the signal-to-noise ratio of the received signals and thereby the estimator performance.
The decrease in data acquisition time opens a window to various applications. As mentioned, deeper lying structures can be imaged at higher frame rates. Another possibility is to use $M-1$ frequency bands for $M-1$ simultaneous range gates for spectral Doppler while using one band for CFM data, as shown in Section IV-B. The authors have named this method multi-frequency quadroplex (MFQ) imaging when using three frequency bands. This can, for instance, be used for investigating the velocity distribution upstream and downstream from an arterial stenosis. Since the upstream and downstream velocities are measured simultaneously, this offers the possibility of deriving relative measures of, for instance, the degree of a stenosis. Furthermore, the use of multiple frequency bands can potentially be used for recognizing aliasing, due to the fact that different frequency bands will alias differently. Thereby aliasing could potentially be compensated for, resulting in larger velocity ranges of the estimator.

The parallel receive beamforming approach presented in [9] was restricted to beamforming closely spaced lines within the transmit beam width. The approach presented in this paper gives full flexibility of which lines are sampled, since the data acquired in different frequency bands are totally independent. However, the presented method, in contrast to the method in [9], can be used only for sampling within a narrow frequency band, and is therefore appropriate only for narrow-band applications such as velocity estimation, and not, for instance, for B-mode imaging. The method has its main advantages in applications such as MFQ imaging, where the data must be independent. Nevertheless, the two methods do not exclude each other. By combining the two methods and sampling $K$ parallel receive lines within each of the $M$ frequency bands, the data acquisition time will decrease by a factor of $K M$. At present, CFMs of three-dimensional volumes make use of ECG-gating for achieving a sufficient frame rate. The modality is thereby strictly speaking not realtime. By combining the two methods and decreasing the data acquisition time by a factor of $K M$, CFMs of threedimensional volumes in real-time become feasible.

\section{ACKnowledgments}

MD Kristoffer Lindskov Hansen is acknowledged for performing the in vivo data acquisition.

\section{REFERENCES}

[1] C. Kasai, K. Namekawa, A. Koyano, and R. Omoto, "Real-time two-dimensional blood flow imaging using an autocorrelation technique," IEEE Trans. Sonics Ultrason., vol. 32, pp. 458-463, 1985.

[2] J. A. Jensen, Estimation of Blood Velocities Using Ultrasound: A Signal Processing Approach. New York: Cambridge University Press, 1996.

[3] O. Bonnefous and P. Pesqué, "Time domain formulation of pulse-Doppler ultrasound and blood velocity estimation by cross-correlation," Ultrason. Imag., vol. 8, pp. 73-85, 1986. 
[4] K. W. Ferrara and V. R. Algazi, "A new wideband spread target maximum likelihood estimator for blood velocity estimationPart I: Theory," IEEE Trans. Ultrason., Ferroelect., Freq. Contr., vol. 38, pp. 1-16, 1991.

[5] L. S. Wilson, "Description of broad-band pulsed Doppler ultrasound processing using the two-dimensional Fourier transform," Ultrason. Imag., vol. 13, pp. 301-315, 1991.

[6] H. Torp and K. Kristoffersen, "Velocity matched spectrum analysis: A new method for suppressing velocity ambiguity in pulsedwave Doppler," Ultrasound Med. Biol., vol. 21, no. 7, pp. 937944, 1995.

[7] P. R. Phillips, "Medical diagnostic ultrasound method and apparatus for improving Doppler processing," U.S. Patent 6179 781, Jan. 30, 2001.

[8] J. Udesen, F. Gran, and J. A. Jensen, "A frequency splitting method for CFM imaging," in Proc. IEEE Ultrason. Symp., 2006, pp. 2019-2022.

[9] D. P. Shattuck, M. D. Weinshenker, S. W. Smith, and O. T. von Ramm, "Explososcan: A parallel processing technique for high speed ultrasound imaging with linear phased arrays," J. Acoust. Soc. Amer., vol. 75, pp. 1273-1282, 1984.

[10] J. W. Allison, "Parallel Doppler processing in ultrasonic flow imaging," Ph.D. thesis, Duke University, Durham, NC, 1987.

[11] Food and Drug Admin., "Information for manufacturers seeking marketing clearance of diagostic ultrasound systems and transducers," Center for Devices and Radiolog. Health, U.S. Food and Drug Admin., Rockville, MD., Tech. Rep., 1997.

[12] F. Gran and J. A. Jensen, "Frequency division transmission and synthetic aperture reconstruction," IEEE Trans. Ultrason., Ferroelect., Freq. Contr., vol. 53, no. 5, pp. 900-911, 2006.

[13] F. Gran and J. A. Jensen, "Directional velocity estimation using a spatio-temporal encoding technique based on frequency division for synthetic transmit aperture ultrasound," IEEE Trans. Ultrason., Ferroelect., Freq. Contr., vol. 53, no. 7, pp. 1289-1299, 2006.

[14] T. Loupas, J. T. Powers, and R. W. Gill, "An axial velocity estimator for ultrasound blood flow imaging, based on a full evaluation of the Doppler equation by means of a two-dimensional autocorrelation approach," IEEE Trans. Ultrason., Ferroelect., Freq. Contr., vol. 42, pp. 672-688, 1995.

[15] J. H. McClellan, T. W. Parks, and L. R. Rabiner, "A computer program for designing optimum FIR linear phase digital filters," IEEE Trans. Audio Electroacoust., vol. AU-21, no. 6, pp. 506-526, 1973.

[16] J. G. Proakis and D. G. Manolakis, Digital Signal Processing, Principles, Algorithms, and Applications. 3rd ed. Upper Saddle River, NJ: Prentice-Hall, 1996.

[17] J. A. Jensen, O. Holm, L. J. Jensen, H. Bendsen, S. I. Nikolov, B. G. Tomov, P. Munk, M. Hansen, K. Salomonsen, J. Hansen, K. Gormsen, H. M. Pedersen, and K. L. Gammelmark, "Ultrasound research scanner for real-time synthetic aperture image acquistion," IEEE Trans. Ultrason., Ferroelect., Freq. Contr., vol. 52, no. 5, pp. 881-891, May 2005.

[18] K. Kristoffersen and B. A. J. Angelsen, "A time-shared ultrasound Doppler measurement and 2-D imaging system," IEEE Trans. Biomed. Eng., vol. BME-35, pp. 285-295, 1988.

[19] J. A. Jensen, "Spectral velocity estimation in ultrasound using sparse data sets," J. Acoust. Soc. Amer., vol. 120, no. 1, pp. 211-220, 2006.

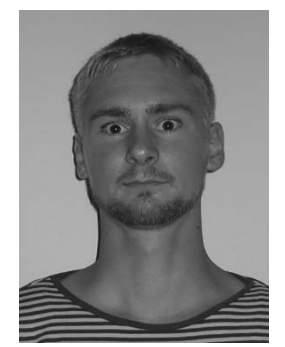

Niels Oddershede was born in Thisted, Denmark, in 1979. He received the M.Sc. degree in electronical engineering from the Technical University of Denmark in 2005, and in 2008 he finished his Ph.D. studies also at the Technical University of Denmark. Since January 2008 he has been employed as a DSP Software Developer at GN ReSound A/S.

His research interests include signal processing with applications in medical ultrasound imaging and real-time audio.

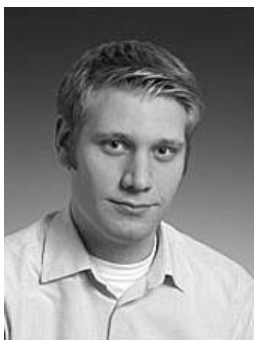

Fredrik Gran earned his M.Sc. in engineering physics from Lund University in 2002. In 2005 Dr. Gran received the Ph.D. from the Technical University of Denmark for work on ultrasound signal processing. From 2005 to 2008 he was employed as Assistant Professor at the Technical University of Denmark. In Jan. 2008, Dr. Gran joined GN ReSound A/S as a research scientist in hearing aid signal processing.

His research interests include adaptive signal processing, adaptive beamforming, and acoustics.

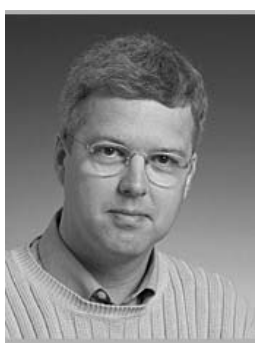

Jørgen Arendt Jensen (M'93-SM'02) earned his M.S. degree in electrical engineering in 1985 and the Ph.D. degree in 1989, both from the Technical University of Denmark. He received the Dr.Techn. degree from the university in 1996. He has published more than 140 journal and conference papers on signal processing and medical ultrasound and the book, Estimation of Blood Velocities Using Ultrasound, Cambridge University Press, in 1996. He is also developer of the Field II simulation program. He has been a visiting scientist at Duke University, Stanford University, and the University of Illinois at Urbana-Champaign. He is currently full professor of Biomedical Signal Processing at the Technical University of Denmark at Ørsted•DTU and head of Center for Fast Ultrasound Imaging. He is also adjunct full professor at the Faculty of Health Sciences at the University of Copenhagen. He has given courses on blood velocity estimation at both Duke University and the University of Illinois and teaches biomedical signal processing and medical imaging at the Technical University of Denmark. He has given several short courses on simulation, synthetic aperture imaging, and flow estimation at international scientific conferences. He has received several awards for his research. He is also the co-organizer of a new biomedical engineering education program offered by the Technical University of Denmark and the University of Copenhagen. His research is centered around simulation of ultrasound imaging, synthetic aperture imaging and blood flow estimation, and constructing systems for such imaging. 